\title{
A Comparative Study on the Bilateral Areolar Endoscopic Approach Versus the Conventional Open Approach for Thyroidectomy
}

xiao ma ( $\square$ madaxiao@qq.com )

Beijing Cancer Hospital

Qi-Jun Xia

PLA Rocket General Hospital

\section{Yun-Tao Song}

Beijing Cancer hospital

Tian-Xiao Wang

Beijing Cancer hospital

\section{Research Article}

Keywords: Thyroidectomy, Bilateral areolar endoscopic approach, Conventional open approach, Visual analog scale

Posted Date: June 7th, 2021

DOl: https://doi.org/10.21203/rs.3.rs-567435/v1

License: (c) (i) This work is licensed under a Creative Commons Attribution 4.0 International License. Read Full License 


\section{Abstract}

Background: To compare the safety, advantages and disadvantages of thyroidectomy for differentiated thyroid carcinoma (DTC) via the bilateral areolar endoscopic approach (BAA) and the conventional open approach (COA).

Methods: Eighty-six female patients who underwent thyroidectomy were enrolled and divided into two groups, 42 in the BAA group and 44 in the COA group, according to aesthetic requirements. The operating time, blood loss during surgery, number of dissected lymph nodes, length of hospital stay, drainage removal time and surgical complications between the two surgical approaches were compared. The pain score and cosmetic results of operative incisions were evaluated with the visual analog scale (VAS).

Results: Patients in the BAA group had significantly less intraoperative blood loss and a significantly shorter scar length $(P<0.001)$ than those in the COA group. However, the operation time of 97.6 minutes in the BAA group was significantly longer than that in the COA group (76.4 minutes; $P<0.001$ ). Comparison of the number of central lymph nodes (CLNs) dissected, drainage removal time and length of hospital stay revealed no significant differences $(P>0.05)$. Patients in the BAA group experienced significantly less pain than those in the COA group $(P<0.001)$. Similarly, patients were significantly more satisfied with the cosmetic outcomes resulting from the BAA than from the COA $(P<0.001)$.

Conclusions: These results show that BAA thyroidectomy exhibits superior advantages in clinical outcomes, such as causing less pain and achieving better cosmetic satisfaction, compared with COA thyroidectomy. Therefore, BAA thyroidectomy is a safe and ideal surgical method for DTC.

\section{Background}

In recent years, the incidence of thyroid carcinoma, especially differentiated thyroid carcinoma (DTC), has been increasing. ${ }^{1,2}$ The prognosis of DTC is excellent, and patients can survive for more than 10 years and even up to 30 years in some cases. ${ }^{3}$ Such disease is very common in young women. Conventional open thyroidectomy is considered extremely safe and remains the most popular method. However, this procedure often leaves an ugly scar on the anterior neck and seriously affects the aesthetic appearance.

Since the first introduction of endoscopic parathyroidectomy by Gagner in 1996 and subsequent report on total endoscopic thyroidectomy by lkeda et al., ${ }^{4-7}$ scarless incisions made possible by the roboticassisted transaxillary approach, video-assisted anterior chest approach and transoral endoscopic approach have been reported to achieve cosmetic satisfaction. However, these innovative procedures are associated with an increased operative time, a need for additional endoscopic instrumentation, new complications (e.g., injury to the brachial plexus and external and internal jugular veins), and hypercapnia. Therefore, some scholars believe that such surgery is an extremely invasive operation rather than a minimally invasive operation. ${ }^{8}$ 
In the present study, we examined the advantages and disadvantages of the bilateral areolar endoscopic approach (BAA) by comparing its clinical effects with those of the conventional open approach (COA). Therefore, the aim of this study was to evaluate and compare the surgical outcomes and aesthetic effects of different procedures in patients with DTC.

\section{Methods}

\section{Patient characteristics and data collection}

We conducted a retrospective analysis in patients with DTC at the Department of Head and Neck Surgery in Perking University Cancer Hospital. A total of 86 female patients who underwent surgical treatment for DTC were enrolled in the current study between March 2017 and December 2017. All patients were diagnosed with DTC through preoperative fine needle aspiration biopsy pathology. Patients were assigned to the BAA group $(n=42)$ and the COA group $(n=44)$ according to their cosmetic requirements. Lobectomy plus ipsilateral central lymph node dissection (CLND) was performed in each patient. The DTC stage ${ }^{9}$ was T1NOM0-T2N1aM0. We retrieved the patients' information, including age, incision length, surgical approach, incidence of complications, pain score and cosmetic assessment, from their medical records. Patients with other medical diseases, such as diabetes or obesity, a smoking history, a keloid tendency, a history of radiotherapy to the head and neck, and incomplete information, were excluded. Recurrent laryngeal nerve (RLN) function was evaluated by electronic fiber laryngoscopy at 1 and 3 months postoperatively. The follow-up time was 3 months. The current study was reviewed and approved by the Ethics Committee of Peking University Cancer Hospital, and informed consent was obtained from all patients to publish the information/image(s) as an online open-access publication. This study was an open-label study, with no blinding of patients, clinicians, or research staff.

\section{Surgical Procedure}

Lobectomy plus CLND was performed by the same surgical team. Patients were divided into the COA group and the BAA group.

$\mathrm{COA}$

A 4 to $5 \mathrm{~cm}$ collar incision was created. Subplatysmal flaps were raised, and the strap muscles were mobilized. Then, the superior pole of the thyroid gland was exposed. Using blunt dissection, the superior pole vessels were isolated and then processed by an ultrasonic coagulation device (Harmonic Scalpel, HS; Ethicon Endosurgery, USA). The parathyroid glands and the RLN were identified and preserved. Then, the gland was delivered through the surgical incision. Finally, CLND was performed. A suction drainage system was used routinely. For specific methods, please refer to previous literature (Fig. 1). ${ }^{10}$

\section{BAA thyroidectomy}


The patients were placed in the supine position with the neck slightly extended. The patients' legs were spread apart, and the surgeon stood between them while wearing a head-mounted monitor. We devised three-port bilateral areolar endoscopic incisions: two $10 \mathrm{~mm}$ incisions (one in the left areola edge: direction, 10-11 o'clock; the other in the right areola edge: direction, 2-4 o'clock) and one $5 \mathrm{~mm}$ incision in the right areola edge (direction, 11-12 o'clock). First, inflation fluid ( $500 \mathrm{ml}$ of normal saline $+0.5 \mathrm{mg}$ of epinephrine) was injected between the deep layer and superficial layer of the anterior chest to reduce bleeding events. No more than 40 milliliters of inflation fluid was injected into each surgical tunnel. A 14$\mathrm{cm}$-long curved Mayo dissecting scissor was used to establish the operating space. Unnecessary inflation fluid was cleared with gauze. A $30^{\circ}$ endoscope was imbedded in the 10-mm punctured sheath in the right mammary areola (direction, 2-4 o'clock), and $\mathrm{CO}_{2}$ was injected to maintain a pressure of $6-8 \mathrm{mmHg}$ (Fig. 2).

With the guidance of an endoscope, the puncture sheath and operating apparatus were imbedded in the other two incisions. An electrical hook was initially employed to separate the subcutaneous loose connective tissue, and sequentially, a work space was built with the outer margins of both sides of the sternocleidomastoid, upper boundary of thyroid cartilage, superficial layer of the broad cervical muscle and deep layer of the anterior cervical band muscle. An ultrasonic scalpel was applied when there were branches of the anterior jugular veins in the middle line. Subsequently, the midline between the genuine and fake thyroid envelopes was opened. The strap muscles were pulled with a special thyroid retractor to reveal the thyroid. The isthmus of the thyroid gland was then removed. First, the pretracheal fascia and suspensory ligaments of the thyroid were disassociated upward and divided until the cricothyroid interval was revealed. All branches of the superior thyroid artery were coagulated carefully, and the superior pole was dissected. Next, the thyroid was pulled towards the inside, and the thyroid veins were cut. Then, the lower pole was lifted, and the inferior blood vessels of the thyroid were handled. Finally, the RLN and the lower parathyroid gland were carefully distinguished. The magnification of the endoscope reduced the difficulty of recognizing the RLN and lower parathyroid gland. The RLN was protected with blue saline gauze strips and traced to its entrance into the cricothyroid junction with division of the ligament of Berry. Here, the upper parathyroid gland needed identification and cautious preservation. Finally, CLND was performed. The specimen was removed from the left bilateral areolar incision and placed in a sterile bag (Fig. 3).

After the suction drainage system was used, interrupted sutures of 5 - 0 Vicryl were used to reapproximate the subcutaneous tissues. The epidermis was fixed with $3 \mathrm{M}$ Steri-Strip elastic skin closures. The chest wall was compressed with an elastic bandage for 24 hours.

\section{Evaluation tool}

Scar length, operative time, intraoperative blood loss, length of hospital stay, drainage removal time and incidence of complications between the two groups were observed. At the same time, the pain score and cosmetic outcome of the incisions were evaluated by using the visual analog scale (VAS) 24 hours and three months after surgery, respectively. On a rating scale of $0-10,0$ indicates no pain, and 10 reflects the 
worst pain. Regarding cosmetic satisfaction, 0 indicates no satisfaction, whereas 10 indicates "very satisfied."

\section{Statistical analysis}

The SPSS statistical package (version 22.0; Chicago, IL) was used to analyze the data. For nonparametric data, the differences between and within groups were analyzed by the chi-square test and Fisher's exact test. Values are reported as the mean \pm standard deviation (SD). For continuous variables, intergroup differences were analyzed by the $T$ test. A P value less than 0.05 was considered significant.

\section{Results}

\section{Patient characteristics}

Between March 2017 and December 2017, 86 patients who underwent thyroid carcinoma surgery were enrolled in this study. Patients were divided into the COA group $(n=44)$ and the BAA group $(n=42)$. The age distribution of the whole population ranged from 18 to 47 years, with an average age of 30.5 years in the COA group and 28.9 years in the BAA group, and there were no significant differences between the two groups. The final pathological diagnosis was papillary carcinoma for all patients.

\section{Comparison of perioperative features between the two groups}

The operation time of 129.0 minutes in the BAA group was significantly longer than that in the COA group (79.6 minutes; $P<0.001$ ). The patients in the BAA group had significantly less intraoperative blood loss $(P<0.001)$ and a significantly shorter scar length $(P<0.001)$ than those in the COA group. The length of hospital stay, drainage extubation time and number of CLNs dissected were not significantly different between the two groups (Table 1). 
Table 1

Comparison of perioperative features

\begin{tabular}{|llll|}
\hline Variable & BAA & COA & \multirow{2}{*}{ p value } \\
\cline { 2 - 3 } & $(\mathbf{n}=\mathbf{4 2})$ & $\mathbf{( n = 4 4 )}$ & \\
\hline Age (years) & $28.9 \pm 4.5$ & $30.5 \pm 6.4$ & 0.095 \\
\hline Operation time (min) & $97.6 \pm 13.9$ & $76.4 \pm 12.2$ & $<0.001$ \\
\hline Blood loss (ml) & $15.3 \pm 8.4$ & $30.2 \pm 11.9$ & $<0.001$ \\
\hline Length of hospital stay (days) & $4.9 \pm 0.4$ & $4.8 \pm 0.6$ & 0.473 \\
\hline Drainage removal time (days) & $2.1 \pm 0.7$ & $2.2 \pm 0.8$ & 0.281 \\
\hline Number of CLNs dissected & $3.1 \pm 0.7$ & $3.2 \pm 0.5$ & 0.322 \\
\hline COA: conventional open approach; BAA: bilateral areolar endoscopic approach.
\end{tabular}

\section{Comparison of the pain score and cosmetic satisfaction with the VAS}

Our results showed that patients in the BAA group experienced significantly less pain than those in the COA group $(P<0.001)$. Similarly, patients were significantly more satisfied with the cosmetic outcomes resulting from BAA thyroidectomy than from COA thyroidectomy. (Table 2).

Table 2

Comparison of the pain scores and cosmetic satisfaction results

\begin{tabular}{|llllll|}
\hline Variable & BAA $(n=42)$ & \multicolumn{3}{c|}{ COA $(n=44)$} & \multirow{2}{*}{$p$ value } \\
\cline { 2 - 5 } & Mean \pm SD & Median (range) & Mean \pm SD & Median (range) & \\
\hline Scar length $(\mathrm{mm})$ & $25.55 \pm 0.34$ & $25(24-26)$ & $43.36 \pm 2.85$ & $44(39-51)$ & $<0.001$ \\
\hline Pain score & $4.13 \pm 2.54$ & $4(3-5)$ & $5.15 \pm 2.44$ & $5(3-7)$ & $<0.001$ \\
\hline Cosmetic satisfaction & $9.52 \pm 1.18$ & $9(8-10)$ & $7.82 \pm 1.76$ & $7(5-8)$ & $<0.001$ \\
\hline COA: conventional open approach; BAA: bilateral areolar endoscopic approach. & \\
\hline $\begin{array}{l}\text { On a rating scale of 0-10, 0 indicates no pain, and 10 indicates the worst pain. Regarding cosmetic } \\
\text { satisfaction, 0 indicates no satisfaction, whereas 10 indicates "very satisfied." }\end{array}$ \\
\hline
\end{tabular}

\section{Complication assessment}

Postoperative complications were compared between the groups. One patient with temporary RLN palsy in the BAA group recovered two months after surgery. No complications were observed in the COA group. No permanent hypocalcemia was reported in any patient. One patient in the COA group developed temporary hypocalcemia, and another patient in the COA group developed a hematoma (Table 3). 
Table 3

Comparison of postoperative complications

\begin{tabular}{|lll|}
\hline Variable & BAA & COA \\
\cline { 2 - 3 } & $\mathbf{( n = 4 2 )}$ & $\mathbf{( n = 4 4 )}$ \\
\hline Temporary RLN palsy & 1 & 0 \\
\hline Permanent RLN palsy & 0 & 0 \\
\hline Temporary hypocalcemia & 0 & 1 \\
\hline Permanent hypocalcemia & 0 & 0 \\
\hline Bleeding & 0 & 0 \\
\hline Hematoma & 0 & 1 \\
\hline Infection & 0 & 0 \\
\hline COA: conventional open approach; BAA: bilateral areolar approach; & \\
\hline RLN: recurrent laryngeal nerve. & \\
\hline
\end{tabular}

\section{Discussion}

In recent years, with the 'inert' characteristic of DTC and the improvement in operation skills, thyroidectomy for DTC has resulted in a good prognosis. However, due to the anatomical position of the thyroid gland, a 6-10 cm surgical scar is usually left in the cervical area after COA thyroid surgery, seriously affecting the cosmetic appearance of the neck. In our study, the BAA approach obtained better cosmetic satisfaction than the COA approach. To meet the cosmetic requirements of the majority of patients, various methods are adopted (e.g., several kinds of concealed incisions and small incisions). Under the guidance of aesthetic principles with the assistance of energy instruments, we improved the cosmetic outcomes by using a shorter collar incision between 3.5 and $4.0 \mathrm{~cm}$ long. Even so, a slight scar could be observed on the anterior neck. ${ }^{10}$

Bilateral areolar endoscopic thyroidectomy could be an ideal approach for thyroid surgery. However, the advantages and benefits of endoscopic thyroid surgery have been controversial because this procedure needs dissection of a large area of subcutaneous tissues, and some new complications may appear, such as bleeding in the tunnel and hypercapnia. The endoscopic apparatus needs a large operating space to reach the thyroid region, which determines the surgical time and surgical injury severity.

Here, we report a simple and easy method to establish the operating space. First, we adopted a low concentration of epinephrine inflation fluid $(500 \mathrm{ml}$ of normal saline $+0.5 \mathrm{mg}$ of epinephrine). Generally, the epinephrine concentration is twice as high. ${ }^{10} \mathrm{In}$ fact, epinephrine inflation fluid at a low concentration may not only shrink the subcutaneous vessel but also avoid subsequent withdrawal bleeding. In our research, no tunnel bleeding was observed with the BAA. Therefore, we recommend this concentration of 
epinephrine inflation fluid. Then, no more than 40 milliliters of inflation fluid was injected into each surgical tunnel, as too much fluid may incur excessive smog when a subsequent energy apparatus is used in the narrow tunnel. After the three incisions were made, we used $14 \mathrm{~cm}$ curved Mayo dissecting scissors to establish the operating space.

The fascia pectoralis divides into two layers to envelop the pectoralis major, and its superficial layer goes beyond the sternum to continue with the contralateral fascia pectoralis. The superficial pectoral fascia (SPF) is easily undermined and separated with a Mayo dissecting scissor. We used a gentle pushing force by the scissor. It was very easy to stretch the SPF in a nonvisual situation. We cut the fiber connective tissue when meeting resistance. The posterior layer of the SPF was dense and difficult to penetrate. We used the other hand to locate the position of the scissor tip to ensure that the scissor was in the correct layer. The time of establishing the operating space in the chest (OSC) was only 3-4 minutes, much less than that with any traditional method. The overall operation time is greatly shortened. We established a small operating space just enough to accommodate the trocar. It was not necessary to dissect the chest flap extensively, so the technique is minimally invasive.

Usually, surgeons utilize a visible flap dissection stick to establish the operating space. ${ }^{11}$ This is a blunt separation process that leads to trauma and rupture of fat cells. Moreover, it needs a long time and a large space, resulting in an extended total operation time and significant surgical injury. Subcutaneous emphysema, fat liquefaction and tunnel bleeding are common complications associated with the BAA but did not occur in our study.

As shown in our results, compared with the COA, the operating time of the BAA was longer. This result is not difficult to comprehend because it is easier to operate in an open incision. However, we thought it was worthwhile for aesthetic purposes at a cost of approximately 30 minutes per operation. We believed we could shorten the operation time with the improvement in skill and accumulation of experience. The number of lymph nodes dissected, postoperative hospitalization time and incidence of postoperative complications were not significantly different between the two groups. The results showed that the BAA technique was safe and reliable. The reduced intraoperative bleeding associated with the BAA also showed the superiority of this technique. In some cases, we were able to finish the operation without any hemostatic gauze.

Furthermore, the BAA group reported lower pain scores than the COA group. Some researchers believe that this could be because the BAA exploits only the subcutaneous tunnels and the neck platysma does not need to be removed. The skin and subcutaneous tissue were well combined when the trocar was extracted. However, in the COA, the neck platysma needs to be removed to expose the thyroid bed. Therefore, the COA was associated with higher pain scores.

Of note, patients in the BAA group reported significantly higher cosmetic satisfaction scores (with a shorter incision length) than patients in the COA group. These data indicate that BAA thyroidectomy is a highly feasible and safe surgical procedure, especially for young female patients. ${ }^{12}$ Nevertheless, our 
current study had some limitations, such as a small sample size, and all patients were derived from a single center. Thus, a large-scale, prospective, multicenter clinical study should be conducted to validate these findings.

\section{Conclusions}

In summary, compared with COA thyroidectomy, BAA thyroidectomy is more desirable in terms of its minimal invasiveness and cosmetic outcomes. It is essential to establish the operating space rapidly and simply. We believe that with the accumulation of surgical experience and continuous improvements in endoscopic instruments, BAA thyroidectomy will be widely used and can be performed for the treatment of more complex thyroid diseases in the future. However, a large-scale, prospective, multicenter clinical study should be conducted to validate the superiority of the BAA.

\section{Abbreviations}

DTC

differentiated thyroid carcinoma

BAA

bilateral areolar endoscopic approach

COA

conventional open approach

VAS

visual analog scale

SPF

superficial pectoral fascia

OSC

operating space in the chest

RLN

recurrent laryngeal nerve

\section{Declarations}

\section{Ethics approval and consent to participate}

This research was reviewed and approved by the Ethics Committee of Peking University Cancer Hospital. All procedures performed in the study involving human participants were in accordance with the ethical standards of the Peking University Cancer Hospital and/or national research committee and with the 1964 Helsinki Declaration and its later amendments or comparable ethical standards.

\section{Consent for publication}

It is unavailable. 


\section{Availability of data and materials}

The datasets used or analyzed during the current study are available from the corresponding author on reasonable request.

\section{Competing interests}

None

\section{Funding}

This research was supported by Science Foundation of Peking University Cancer Hospital 202003.

\section{Authors' contributions}

Xiao Ma developed the concept of the article. Xiao Ma, Yun-Tao Song and Tian-Xiao Wang developed the design and methodology. Xiao Ma, Qi-Jun Xia, and Tian-Xiao Wang contributed to the drafting of the manuscript. All authors read and approved the final manuscript.

\section{Acknowledgements}

The authors thank patients, faculty, and staff in the Departments of Head and Neck at Perking University Cancer Hospital and the Departments of General Surgery at PLA Rocket General Hospital for their participation in patient care and editing the manuscript.

\section{References}

1. Davies L, Welch HG. Current thyroid cancer trends in the United States. JAMA Otolaryngol Head Neck Surg. 2014;140:317-22.

2. Leenhardt L, Grosclaude P, Cherie-Challine L. Increased incidence of thyroid carcinoma in france: a true epidemic or thyroid nodule management effects? Report from the French thyroid cancer committee. Thyroid. 2004;14:1056-60.

3. Mitchell I, Livingston EH, Chang AY, et al. Trends in thyroid cancer demographics and surgical therapy in the United States. Surgery. 2007;142:823-8. discussion 828.e821.

4. Gagner M. Endoscopic subtotal parathyroidectomy in patients with primary hyperparathyroidism. $\mathrm{Br}$ J Surg. 1996;83:875.

5. Huscher CS, Chiodini S, Napolitano C, Recher A. Endoscopic right thyroid lobectomy. Surg Endosc. 1997;11:877.

6. Wang C, Zhai H, Liu W, et al. Thyroidectomy: a novel endoscopic oral vestibular approach. Surgery. 2014;155:33-8.

7. Mitchem JB, Gillanders WE. Endoscopic and robotic thyroidectomy for cancer. Surg Oncol Clin N Am. 2013;22:1-13, v. 
8. Dralle $\mathrm{H}$, Machens A, Thanh PN. Minimally invasive compared with conventional thyroidectomy for nodular goitre. Best Pract Res Clin Endocrinol Metab. 2014;28:589-99.

9. Haugen BR, Alexander EK, Bible KC, et al. 2015 American thyroid association management guidelines for adult patients with thyroid nodules and differentiated thyroid cancer: the American thyroid association guidelines task force on thyroid nodules and differentiated thyroid cancer. Thyroid. 2016;26:1-133.

10. Ma X, Xia QJ, Li G, Wang TX, Li Q. Aesthetic principles access thyroidectomy produces the best cosmetic outcomes as assessed using the patient and observer scar assessment scale. BMC Cancer. 2017;17:654.

11. Yang $C$, Wang Y. A novel surgery technique: non-visual dissection for establishing the operating space during total endoscopic thyroidectomy. Surg Endosc. 2017;31:5451-6.

12. Choi JY, Lee KE, Chung KW, et al. Endoscopic thyroidectomy via bilateral axillo-breast approach (BABA): review of 512 cases in a single institute. Surg Endosc. 2012;26:948-55.

\section{Figures}
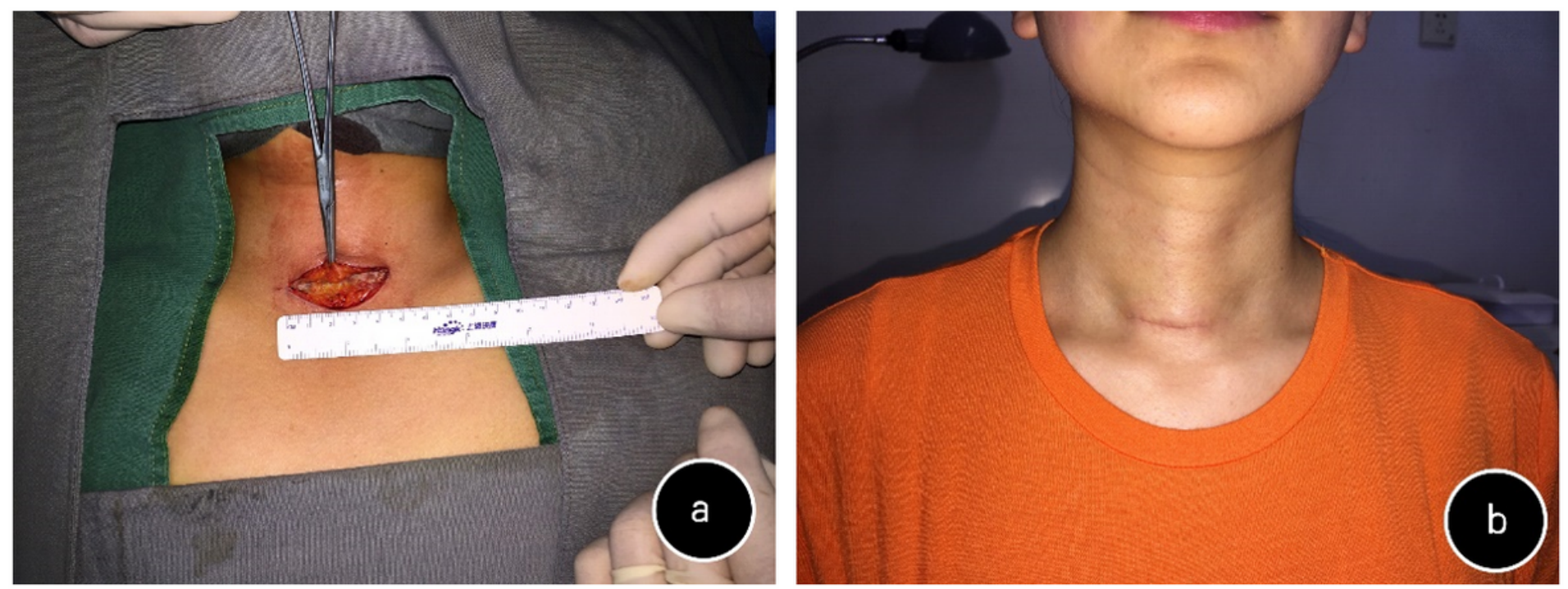

\section{Figure 1}

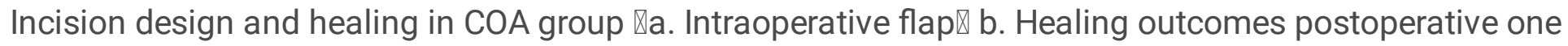
year》 

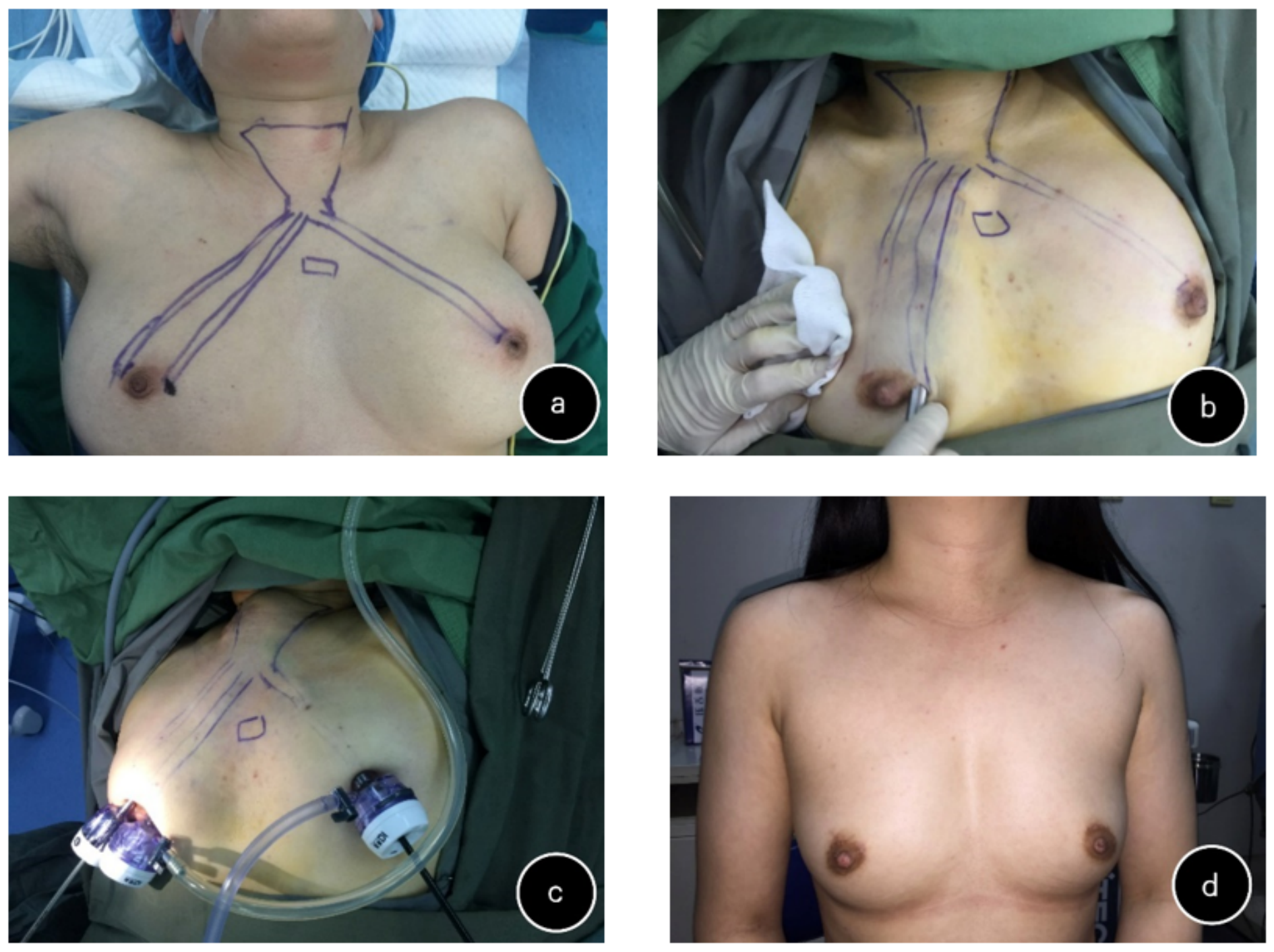

Figure 2

Surgical procedure of BAA group $\mathrm{a} \triangle$ Patient position and port placement with the BAA $\mathrm{b}$. Making the operation space with a Mayo dissecting scissor $\mathrm{c}$. Three incisions were made, and trocars were inserted d. Postoperative outcomes of the BAA
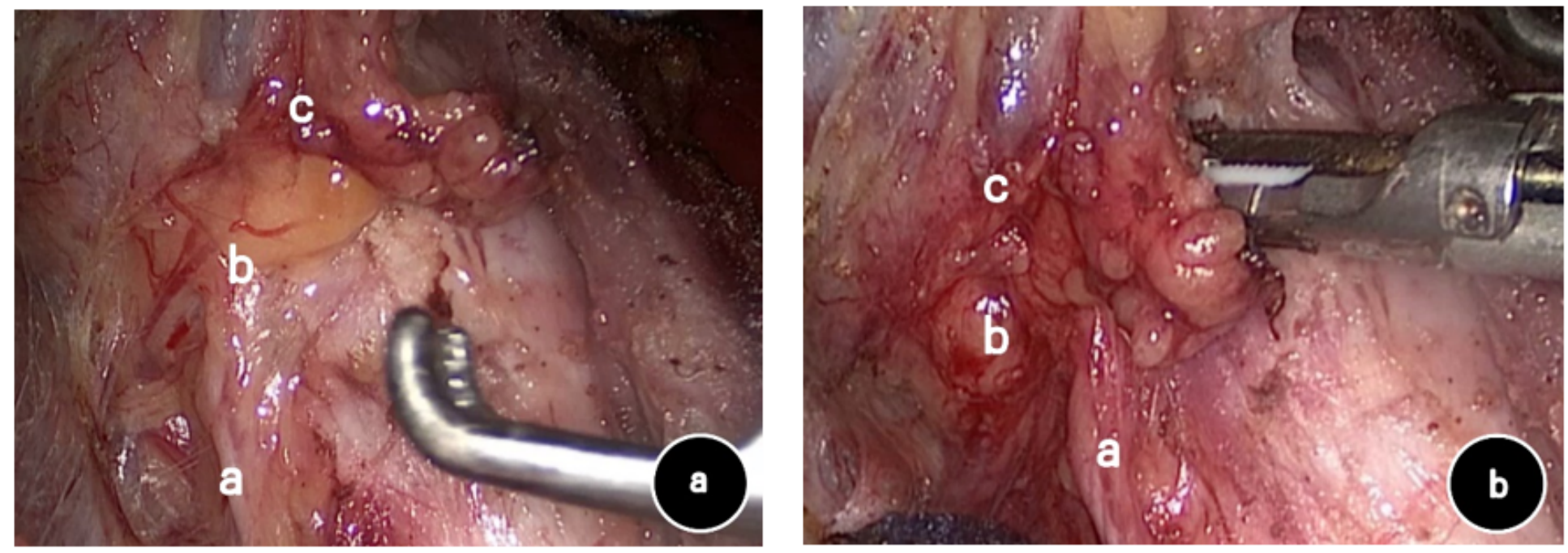


\section{Figure 3}

Endoscopic structure of BAA a. a: RLN b: Inferior parathyroid gland c: Thyroid inferior pole b. a: RLN b: Superior parathyroid gland c: Upper pole of thyroid lobe 